\title{
Dimensionamento da frota de navios de derivados claros para cabotagem: proposta de modelo de otimização
}

\author{
André Augusto Soares Vieira ${ }^{1}$, Silvio Hamacher ${ }^{2}$, luri Martins Santos ${ }^{3}$ \\ ${ }_{1}^{1}$ Departamento de Engenharia Industrial, Pontifícia Universidade Católica do Rio de Janeiro, andre.vieira@petrobras.com.br \\ 2Departamento de Engenharia Industrial, Pontifícia Universidade Católica do Rio de Janeiro, hamacher@puc-rio.br \\ ${ }^{3}$ Departamento de Engenharia Industrial, Pontifícia Universidade Católica do Rio de Janeiro, iuri.santos@tecgraf.puc-rio.br
}

\section{Recebido:}

01 de fevereiro de 2017

Aceito para publicação:

03 de outubro de 2017

Publicado:

29 de outubro 2017

Editor de área:

Marcio D'Agosto

\section{Palavras-chaves:}

Dimensionamento de frota de navios,

Transporte marítimo,

Logística do petróleo,

Transporte industrial.

\section{Keywords:}

Maritime fleet size and mix,

Maritime transportation,

Oil \& gas logistics,

Industrial shipping.

DOI:10.14295/transportes.v25i3.1315

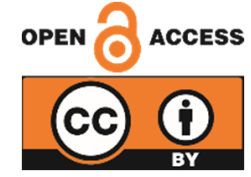

\begin{abstract}
RESUMO
O setor de Óleo e Gás depende fortemente do transporte marítimo para movimentar grandes volumes de petróleo e derivados. Neste sentido, este artigo propõe um modelo de programação linear inteiro misto cujo objetivo é dimensionar o tamanho da frota e o porte dos navios para o transporte de derivados claros restritos à navegação de cabotagem de uma empresa real. O modelo proposto auxiliou os programadores a definir quantos navios são realmente necessários para atender os compromissos assumidos dentro de um horizonte de planejamento pré-estabelecido. Comparando este resultado com o número de navios já contratados e disponíveis, pode-se, eventualmente, liberar navios para realizar viagens de longo curso, evitando assim a contratação de navios em Voyage Charter Party para este fim, além de reduzir a sobre-estadia nos períodos de baixa movimentação, quando a frota contratada se torna superdimensionada.
\end{abstract}

\begin{abstract}
The Oil \& Gas sector relies mainly on the maritime transport to ship large volumes of petroleum and products. In this sense, this paper proposes a mixed integer linear model whose objective is to determine the coastal navigation fleet size and the type of vessels to transport clean petroleum products of real company. The model's outcome helped programmers define how many ships are actually required to meet the commitments undertaken within a pre-established planning horizon. Comparing the model's result with the actual number of vessels available for programming, one can eventually release ships to make other journeys, thus avoiding the hiring of ships in Voyage Charter Party for this last purpose. It is also possible to reduce the demurrage in periods of low movement, when the fleet contracted becomes oversized.
\end{abstract}

\section{INTRODUÇÃO}

O transporte marítimo ocupa um lugar de destaque no comércio internacional, movimentando o maior volume de produto, por longas distâncias, ao menor custo (UNCTAD, 2016). No Brasil, o transporte marítimo, em especial a cabotagem, desempenha um importante papel na atividade econômica brasileira devido a longa faixa litorânea nacional. Dados referentes a 2016 registraram um movimento de 211,8 milhões de toneladas entre os portos brasileiros nesse ano (CNT, 2016). Devido ao seu valor econômico, o transporte marítimo está atraindo cada vez mais a atenção de pesquisadores e gestores empresariais. Segundo Christiansen et al. (2013), a publicação de estudos e pesquisas sobre este tema tem dobrado a cada década. Uma parcela destes estudos visa o dimensionamento da frota de navios de maneira adequada e eficiente. De acordo com Pantuso et al. (2013), empresas de navegação operam em um ambiente 
mutável e incerto, onde o dimensionamento ótimo da frota consiste em uma decisão estratégica crucial. Christiansen et al. (2007) afirmaram que o planejamento adequado das frotas e de suas operações tem o potencial de melhorar o desempenho econômico e reduzir os custos de transporte, sendo este um dos principais desafios enfrentados pelos atores da indústria a fim de se manterem competitivos.

Este tipo de problema é inerente às atividades da empresa de petróleo estudada (uma multinacional brasileira com diversas refinarias, terminais, dutos e navios). Para transportar toda a produção de petróleo e atender o mercado nacional de derivados, ela conta com uma frota de petroleiros próprios e contratados com portes e capacidades variadas que operam em portos com restrições diversas. A empresa revisa anualmente o dimensionamento desta frota, indicando as alterações necessárias para torná-la aderente às demandas futuras de transporte. Atualmente, este dimensionamento é realizado de maneira empírica, sem ferramentas de otimização ou de simulação. Uma vez dimensionada, a frota é colocada à disposição dos programadores de navios para alocá-los da melhor maneira possível. No entanto, devido às incertezas existentes no ambiente de programação, avaliar a aderência da frota dimensionada à real necessidade de transporte torna-se uma difícil tarefa. No intuito de auxiliar os programadores, este artigo tem como objetivo propor um modelo matemático de programação linear inteiro misto (PLIM) para dimensionar a frota de navios de derivados claros para a cabotagem da empresa, buscando minimizar os custos totais da frota dimensionada, em um horizonte de planejamento pré-estabelecido. Este modelo é uma extensão do modelo proposto por Steffensen (2012) que, além de dimensionar o tamanho da frota, também indica o porte de cada um dos navios que a compõe.

Este artigo está organizado em seis seções, sendo a primeira esta introdutória. A seção 2 apresenta uma revisão bibliográfica sobre os problemas de programação de navios e de dimensionamento de frota. A seção 3 expõe o contexto logístico no qual a companhia está inserida atualmente. A seção 4 descreve o modelo proposto, assim como as simplificações que se fizeram necessárias para o seu desenvolvimento. A seção 5 descreve o estudo de caso, onde os resultados do modelo serão apresentados, e realiza uma análise de cenários, com o intuito de verificar a aderência do modelo quando exposto a situações adversas. Por fim, a seção 6 apresenta as conclusões e as recomendações para futuras pesquisas.

\section{REVISÃO DA LITERATURA}

No intuito de ressaltar a importância da programação de navios, esta seção oferece uma revisão da literatura sobre este tema, e quais são as principais soluções elaboradas pela comunidade científica. A seção 2.1 apresenta quais são os principais problemas em programação de navios. A seção 2.2 traz um aprofundamento sobre os aspectos importantes dos conceitos envolvidos no dimensionamento da frota e dos tipos de navios. A seção 2.3 descreve os níveis de planejamento do abastecimento do petróleo.

\subsection{Problemas em programação de navios}

Segundo Christiansen et al. (2007) e Steffensen (2012), os problemas em programação de navios podem ser classificados, de acordo com o horizonte de planejamento, em estratégicos (que se estendem por vários anos), táticos (por meses) e operacionais (decisões do dia a dia). Entre os problemas estratégicos, estão: seleção de mercado e do comércio; projeto de navios; projeto de sistema de rede e transporte (incluindo a determinação de pontos de transbordo de serviços intermodais); dimensionamento de frota e dos tipos de navios; e projetos de portos e terminais. Já os problemas táticos incluem: ajustes no tamanho e tipo da frota; fleet deployment (atribuição de navios específicos a rotas comerciais); roteamento e programação de navios; programação de navios associado à gestão de estoque nos pontos de carga e descarga; sequenciamento de atracações em berços; sequenciamento de utilização de guindastes; gerenciamento de pátio de contêineres; planejamento de armazenagem de contêineres; gestão de navios; e distribuição de contêineres vazios. Por último, os problemas operacionais envolvem: seleção de velocidade de cruzeiro; carregamento de navios; e roteamento sujeito a restrições ambientais e climáticas. Em todas estas classes, a Pesquisa Operacional é largamente aplicada para encontrar a melhor solução para os principais problemas existentes. 
A primeira revisão da literatura sobre a aplicação da pesquisa operacional em programação de navios foi apresentada por Ronen (1983). Mais recentemente, Ronen (1993), Christiansen et at. (2004) e Christiansen et al. (2013) realizaram outras revisões acadêmicas com um escopo de pesquisa maior, incluindo problemas como dimensionamento de frota e de tipos de navios e gestão de estoque nos portos. As quatro revisões aqui citadas constituem um valioso material para estudo e aprofundamento sobre o emprego da pesquisa operacional em programação de navios. Alguns trabalhos recentes aplicados a programação de navios destacam-se. Diz et al. (2015) propuseram um sistema de suporte à decisão baseado em otimização para a programação de navios de longo curso de petróleo para a Petrobras. Mulder e Dekker (2014) combinam os problemas de desenho da frota, programação de navios e roteamento nas operações de liner shipping e agregam os portos em clusters para reduzir a complexidade do problema.

\subsection{Dimensionamento da frota e dos tipos de navios}

O dimensionamento da frota e dos tipos de navios consiste em determinar a quantidade e o porte dos navios que deverão compor a frota necessária para atender a todos os compromissos dos armadores ou afretadores dos navios. Recentemente, duas revisões de literatura foram publicadas e, juntas, formam um panorama bastante completo sobre o tema. A primeira foi publicada por Hoff et al. (2010) e apresentou uma visão geral do dimensionamento de frota e do roteamento de veículos. A segunda, publicada por Pantuso et al. (2013), apresentou uma revisão específica sobre o dimensionamento de frota para o transporte marítimo. Os autores classificam os modelos encontrados na literatura em single-period maritime fleet size and mix models e multi-period maritime fleet size and mix models. A primeira categoria é focada no dimensionamento de frota de navios para sistemas de transporte cujas características não se alteram com o passar do tempo. Normalmente aplica-se a operações de curto prazo. A segunda categoria é uma extensão da primeira, onde se busca um ajuste dinâmico da frota em resposta à evolução das necessidades de transporte.

O primeiro artigo no tema, Dantzig e Fulkerson (1954), propôs useram um modelo de programação linear para minimizar o número de navios-tanque necessários para transportar óleo combustível para abastecimento de navios da marinha. Décadas mais tarde, Xinlian et al. (2000) apresentaram um algoritmo que combina técnicas de programação linear com técnicas de programação dinâmica para melhorar a solução de um modelo de dimensionamento de frota previamente apresentado em Xinlian et al. (1989) para planejamento da composição da frota no longo prazo, definindo quais tipos de navios devem ser adicionados ou retirados anualmente de uma frota já existente, a fim de atender um determinado padrão de demanda. Destaca-se também a aplicação de uma heurística de busca tabu por Zeng e Yang (2007) para dimensionar a frota de navios no transporte marítimo de carvão e otimizar o roteamento dos mesmos.

Alguns autores tratam de problemas de horizontes mais longos, com maior incerteza, utilizando otimização estocástica ou robusta. Destes, destacam-se dois trabalhos. 0 primeiro, Bakkehaug et al. (2014), propôs um modelo para renovação estratégica da frota com programação estocástica em multi-estágios ( 3 fases no caso). 0 modelo proposto trabalha explicitamente com a incerteza nos parâmetros como demanda futura, taxas de frete e preços dos navios em diferentes horizontes de planejamento e explica as formas de modelar a incerteza. 0 segundo, Gundegjerde et al. (2015), propôs um modelo de programação estocástica com 3 fases - com incertezas nas taxas spot dos navios, nas condições de tempo, no preço da energia elétrica e nas falhas do sistema - para o dimensionamento do tamanho de uma frota de navios e helicópteros que atuam na manutenção e suporte das operações offshore de turbinas eólicas.

Em sua dissertação de mestrado, Steffensen (2012) avaliou a precisão de um modelo matemático já existente para o dimensionamento da frota de navios, desenvolvido pelo Norwegian Marine Technology Research Institute (MARINTEK). 0 autor explorou a aplicação do modelo em uma empresa de navegação do segmento Roll-on-roll-off (Ro-Ro), variando o horizonte de planejamento, o custo do bunker e utilizando variáveis continuas ao invés de variáveis inteiras. Para checar a confiabilidade do modelo, o autor propôs um novo modelo para alocar a frota dimensionada às rotas existentes. 0 modelo investigado pelo 
autor, após algumas modificações, mostrou-se bastante aderente ao problema de dimensionamento de frota dos navios de derivados de petróleo e, por este motivo, foi escolhido para servir de base no estudo de caso. Tanto o estudo de caso como as modificações realizadas serão apresentados na sequência deste trabalho.

\subsection{Planejamento do abastecimento}

Segundo Nunes et al. (2010), o planejamento do abastecimento de petróleo e derivados é dividido em: estratégico, tático, operacional e controle. 0 estratégico consiste no planejamento de longo prazo, realizado através do plano de investimentos. 0 tático engloba o planejamento da comercialização, da alocação de petróleos e do refino, sendo realizado através de uma ferramenta de otimização baseada em programação linear multi-período. 0 planejamento operacional, ou de curto prazo, engloba a programação de navios de cabotagem de petróleos e derivados, assim como o acompanhamento semanal do suprimento e da demanda.

\section{SITUAÇÃO DO DIMENSIONAMENTO DA EMPRESA}

Esta seção apresenta a lógica de planejamento da empresa estudada, e como são realizados atualmente o dimensionamento de frota e a atividade de programação de navios de cabotagem de derivados claros. 0 item 3.1 contextualiza como o dimensionamento de frota é realizado atualmente. 0 item 3.2 caracteriza a programação de cabotagem, onde as restrições operacionais, as prioridades e os princípios que norteiam este processo serão identificados e descritos.

\subsection{Dimensionamento da frota}

0 dimensionamento da frota de navios da empresa é realizado anualmente, mas não há nenhuma ferramenta de otimização para auxiliar este processo. Caso a frota existente seja maior do que a planejada, os contratos de afretamento vigentes não são renovados, caso contrário, contrata-se os navios necessários. É importante ressaltar que esta frota é dimensionada para o período de um ano, onde existem picos e vales de demanda e produção e por isso existe a possibilidade de haver falta ou sobra de navios ao longo do ano.

\subsection{Programação de cabotagem de derivados}

A programação de navios de cabotagem de derivados claros possui dois objetivos principais. 0 primeiro refere-se à necessidade de atender a demanda dos clientes, em volume e tempo adequados. A empresa de petróleo estudada detém aproximadamente 97\% do market-share do mercado de combustíveis do país. Sendo assim, falhas no suprimento podem ocasionar a falta de produto para os consumidores finais. O segundo objetivo é escoar as refinarias de maneira adequada. A redução de carga e a interrupção do processamento de petróleo devido à falta de espaço para armazenamento dos derivados interferem diretamente na rentabilidade da refinaria.

As decisões tomadas na programação de navios de derivados são balizadas por diversas restrições operacionais (restrições dos navios e restrições do próprio terminal) e as condições comerciais existentes entre a empresa e seus clientes. Os programadores analisam diariamente os estoques de cada região e as posições dos navios e realizam o roteamento dos navios, atentando-se para cumprir todos os objetivos de maneira eficiente. As viagens são alocadas a todos os navios da frota de forma a escoar as refinarias e atender o mercado consumidor e as premissas de produção e de mercado utilizadas podem variar com o tempo. A produção pode variar por alterações no elenco de petróleo, falhas ou manutenções emergenciais. 0 mercado também pode variar em função de problemas climáticos, crises econômicas e sazonalidade. Há momentos em que a capacidade de transporte representada por esta quantidade de navios é maior do que a necessária para se cumprir todos os compromissos de movimentação. Nestas ocasiões, alguns navios podem ser alocados em viagens de longo curso. Também pode acontecer a situ- 
ação inversa, onde é necessário afretar um ou mais navios em Voyage Charter Party (VCP, contrato de afretamento por viagem) para complementar a frota. Atualmente, não há nenhuma ferramenta de otimização para auxiliar os programadores da empresa nestas tarefas, o que os obriga a necessitar de intensivos e contínuos treinamentos para aprender a lidar com centenas de variáveis simultaneamente.

Neste sentido, o estudo de caso apresentado no decorrer deste artigo irá propor um modelo de otimização que tem por objetivo dimensionar a frota de navios claros necessários para movimentar um determinado volume de derivados, em um horizonte de tempo pré-estabelecido. Este dimensionamento irá ajudar o programador a verificar se há possibilidade de liberar algum navio da frota para carregar uma carga de importação, evitando assim o afretamento de um navio para realizá-la.

\section{DESCRIÇÃO DO MODELO MATEMÁTICO PROPOSTO}

O modelo matemático de programação linear inteiro misto (PLIM) proposto nesta seção foi baseado no modelo apresentado por Steffensen (2012). Algumas adaptações foram necessárias para torná-lo aderente ao caso estudado. Considerou-se apenas uma velocidade de cruzeiro, diferente do modelo original que permitia selecionar a velocidade de navegação para cada viagem. Restrições de capacidade de produto por tipo de navio, restrições de navios nos portos e restrições de quantidades de viagens mínimas em cada arco foram eliminadas, enquanto que restrições referentes a calado (equações 5 e 6 ) foram introduzidas. Restrições de suprimento (equações 3) e demanda (equações 4) foram modificadas para usar os parâmetros dos nós ao invés dos arcos. No item 4.1, as premissas que se fizeram necessárias para a execução do modelo são detalhadas. Por fim, o item 4.2 apresenta a nomenclatura e o modelo propostos.

\subsection{Premissas utilizadas}

0 problema real de programação de navios está inserido em um ambiente carregado de incertezas, como variações da demanda, problemas operacionais em refinarias, navios, dutos e terminais, preço futuro dos combustíveis, entre outros. Como o modelo aqui proposto pretende resolver um problema de transporte para o curto prazo, alguns aspectos foram simplificados. A primeira premissa é que se trata de um modelo de single-period maritime fleet size and mix problem, aonde as características do ambiente são consideradas previsíveis e constantes ao longo do curto período de planejamento, não havendo incerteza em parâmetros como oferta e demanda dos terminais, preço de aluguel dos navios e custos de combustíveis dos navios (bunker e marine diesel oil). Outra consideração importante é que não se considera nenhum tipo de roteirização, sendo um problema de transporte sem transbordo. Diversos artigos científicos disponíveis na literatura propõem modelos matemáticos para resolver esta questão, no entanto, entendeu-se que, no caso da cabotagem, as viagens são muitas vezes realizadas em ida e volta, o que torna a roteirização desnecessária para o dimensionamento da frota de navios de derivados na cabotagem. Além disso, alguns portos onde a movimentação de derivados claros é inexpressiva comparada com o total movimentado foram omitidos. A utilização deles poderia contaminar os resultados e dificultar a interpretação por parte dos programadores. Outra premissa foi de que a velocidade de navegação seja constante para todos os navios, estabelecendo-se uma velocidade média de 13 nós para todos os navios. Além disso, na cabotagem de derivados claros, é comum o compartilhamento de cargas em uma mesma viagem para economizar em escala, premissa que também foi adotada no modelo utilizado.

Por último, não foi considerado na construção do modelo o gerenciamento de estoques nos portos de origem e destino, conhecido como Maritime Inventory Routing (MIR), que foi definido por Christiansen et al. (2013) como sendo um problema de programação, onde um ator da cadeia de suprimentos possui a responsabilidade pelo gerenciamento dos estoques nos terminais e pela programação e roteamento dos navios, implicando em atualizações diárias de dados e parâmetros, que tornariam o modelo para dimensionamento de frota pouco funcional, uma vez que diariamente haveriam alterações na quantidade ótima de navios da frota. 


\subsection{Nomenclatura utilizada e descrição do modelo proposto}

Segue adiante a nomenclatura utilizada no modelo proposto:

Tabela 1: Conjuntos do modelo proposto

\begin{tabular}{llll}
\hline Cód. & Índice & Nome & Descrição \\
\hline$V$ & $V$ & Tipos de navios & Conjunto dos tipos de navios que irão compor a frota. \\
$N$ & $\{i, j\}$ & Nós & Conjunto de nós que representam os portos de carga e descarga. \\
$P$ & $p$ & Produtos & Conjunto dos produtos a serem transportados. \\
\hline
\end{tabular}

Tabela 2: Parâmetros do modelo proposto

\begin{tabular}{|c|c|c|c|}
\hline Cód. & Nome & Descrição & Unidade \\
\hline$N_{i v}$ & Nós-e-navios capacitados & $\begin{array}{l}\text { Matriz binária indicando os nós que permitem a operação de cada um dos } \\
\text { tipos de navios. }\end{array}$ & - \\
\hline$A_{i j}$ & Arco de movimentação & $\begin{array}{l}\text { Parâmetro binário que define quais são os arcos entre os nós } i \text { e } j \text { onde } \\
\text { existe oferta e demanda para um mesmo produto. }\end{array}$ & - \\
\hline$S_{i p}$ & Suprimento & Suprimento do produto $p$ no nó $i$ & $m^{3}$ \\
\hline$D_{i p}$ & Demanda & Demanda do produto $p$ no nó $i$ & $m^{3}$ \\
\hline$T_{i j}$ & Tempo & Tempo total de viagem e operação entre os nós $i$ e $j$. & dias \\
\hline$H$ & $\begin{array}{l}\text { Horizonte de planeja- } \\
\text { mento }\end{array}$ & Horizonte de planejamento no qual a frota deverá ser dimensionada & dias \\
\hline$C_{v i j}$ & Custo operacional & $\begin{array}{l}\text { Custo formado pelo somatório dos custos de combustível (bunker e diesel) e } \\
\text { das despesas portuárias em cada arco }(i, j) \text { para cada classe de navio. }\end{array}$ & US\$ \\
\hline$C_{v}^{T C P}$ & Custo fixo & Custo diário do contrato Time Charter Party por utilizar o navio do tipo $v$. & US\$/dia \\
\hline$C L_{v i}$ & Calado & $\begin{array}{l}\text { Volume máximo que pode ser carregado ou descarregado no navio do tipo } v \\
\text { no porto } i \text { devido a restrições de calado. }\end{array}$ & $m^{3}$ \\
\hline
\end{tabular}

Tabela 3: Variáveis de decisão do modelo proposto

\begin{tabular}{llll}
\hline Cód. & Descrição & Unidade \\
\hline$x_{v i j}$ & Variável inteira igual ao número de viagens dos navios do tipo $v$ entre os nós $i$ e $j$. & - \\
$y_{v i j p}$ & Quantidade de produto $p$ transportada pelo navio do tipo $v$ entre os nós $i$ e $j$. & $\mathrm{m}^{3}$ & - \\
$z_{v}$ & Variável inteira igual ao número de navios do tipo $v$. & - \\
\hline
\end{tabular}

Para que a variável $X_{v i j}$ seja criada, é necessária a existência do arco entre os nós $i$ e $j$, conforme define o parâmetro $A_{i j}$, e que o navio do tipo $v$ esteja apto a operar em ambos os nós, conforme define o parâmetro $N_{i v}$. Além disso, é necessário que as restrições de calado definidas pelo parâmetro $C L_{v i}$ sejam cumpridas. A criação da variável $y_{v i j p}$ também é restrita pelos parâmetros $A_{i j}$ e $N_{i v}$. Ademais, é necessário limitá-la à demanda do nó $j$, conforme define o parâmetro $D_{i p}$. Após definidos todos os conjuntos, índices, parâmetros e variáveis, o modelo matemático pode ser apresentado. Segue a adiante a função objetivo (Equação 1):

$$
\operatorname{Min} \sum_{i \in N} \sum_{j \in N} \sum_{v \in V} C_{v i j} X_{v i j}+\sum_{v \in V} H C_{v}^{T C P} Z_{v}
$$

Sujeito às seguintes restrições:

$$
\begin{array}{ll}
\sum_{i \in N_{i v}} x_{v i j}=\sum_{i \in N_{i v}} x_{v j i} & \forall v \in V, j \in N_{j v} \\
\sum_{j \in N} \sum_{v \in V} y_{v i j p} \leq S_{i p} & \forall i \in N_{i v}, p \in P \\
\sum_{i \in N} \sum_{v \in V} y_{v i j p}=D_{j p} & \forall j \in N_{j v}, p \in P
\end{array}
$$




$$
\begin{array}{ll}
\sum_{p \in P} y_{v i j p} \leq C L_{v i} X_{v i j} & \forall v \in V, i \in N_{i v} j \in N_{j v} \\
\sum_{p \in P} y_{v i j p} \leq C L_{v j j} X_{v i j} & \forall v \in V, i \in N_{i v} j \in N_{j v} \\
\sum_{(i, j) \in N ; A_{i j}=1} T_{i j} X_{v i j} \leq H z_{v} . & \forall v \in V \\
z_{v} \in \mathbb{Z}_{\geq 0} & \forall v \in V \\
X_{v i j} \in \mathbb{Z}_{\geq 0} & \forall v \in V, i \in N_{i v} j \in N_{j v} \\
y_{v i j p} \geq 0 & \forall v \in V, i \in N_{i v} j \in N_{j v},
\end{array}
$$

A equação (1) é a função objetivo, que busca minimizar os custos totais da frota. 0 primeiro termo da equação (1) representa a somatória dos custos variáveis, composto pelo custo dos combustíveis marítimos e pelas despesas portuárias em função da quantidade de viagens. Como a empresa analisada utiliza o contrato de afretamento por tempo (TCP - Time Charter Party), o custo do frete independe da quantidade transportada, já que a empresa tem de pagar o valor cheio da viagem independentemente do volume. 0 segundo termo da equação (1) representa a somatória dos custos fixos, composto pelo custo do contrato de afretamento no período do horizonte de planejamento. 0 conjunto de restrições (2) garante que todas as viagens iniciem e terminem no mesmo nó $i$. Ou seja, toda viagem é um ciclo completo. As restrições (3) restringem o volume a ser carregado no porto de carga ao volume disponível neste porto durante o horizonte de planejamento. 0 conjunto de restrições (4) garante que a demanda dos clientes nos portos de descarga será integralmente atendida. As equações (5) e (6) restringem o volume a ser carregado nos portos de origem e descarregado nos portos de destino em função das restrições de calado máximo de acordo com o tipo de navio e o porto, já considerando a capacidade da classe do petroleiro. Com isso, o calado máximo é mais restritivo que a capacidade dos navios, o que torna as restrições clássicas de capacidade dos navios desnecessárias. As restrições (7) asseguram que o número de dias de operação para todos os navios de cada porte é menor ou igual ao número de dias disponíveis no horizonte de planejamento. As equações (8), (9) e (10) apresentam o conjunto universo das variáveis.

\section{ESTUDO DE CASO}

Esta seção apresenta os dados de entrada e os resultados do modelo matemático de dimensionamento da frota dos navios claros da empresa estudada. A seção 5.1 apresenta as definições e características de todos os dados de entrada considerados. A seção 5.2 traz uma descrição detalhada e uma análise crítica dos resultados do modelo para diversos cenários.

\subsection{Dados de Entrada}

Para testar a validade do modelo, uma série de dados de entrada e parâmetros iniciais precisa ser levantada para reproduzir o ambiente de programação de navios. Adotando a mesma classificação utilizada por Diz et al. (2015), estes dados e parâmetros foram divididos entre dados fixos e dados dos cenários. Os dados fixos são aqueles que não variam com frequência ao longo do tempo, como por exemplo, porte dos navios, custo operacional, tempo de viagem, custo dos contratos TCP, entre outros. A Tabela 4 descreve as principais classes de navios no estudo de caso. Destaca-se que navios de maior porte como Panamax e Aframax de derivados claros não foram utilizados devido a baixíssima frequência deles na cabotagem brasileira.

Os produtos levados em consideração para a construção do modelo foram: nafta petroquímica, gasolina, gasolina de alta octanagem (booster), querosene de aviação (QAV), diesel S10, diesel $S 500$ e diesel com alto teor de enxofre (ATE). Estes produtos representam cerca de $98 \%$ de toda a movimentação de 
derivados claros da empresa. Analisando as ofertas e demandas na cabotagem, determinaram-se 8 portos de carga e 14 de descarga, sendo alguns portos simultaneamente de carga e descarga, apresentados geograficamente na Figura 1.

Tabela 4: Navios claros na cabotagem (Fonte: autores; Saraceni, 2006; Rocha, 2012; Pelizaro, 2008)

\begin{tabular}{lllllll}
\hline $\begin{array}{l}\text { Classe de } \\
\text { Navio }\end{array}$ & $\begin{array}{l}\text { Calado (draft) } \\
\text { em } \boldsymbol{m}\end{array}$ & $\begin{array}{l}\text { Boca (breadth/ } \\
\text { beam) em } \boldsymbol{m}\end{array}$ & $\begin{array}{l}\text { Comprimento } \\
\text { fora a fora (LOA, } \\
\text { length overall) } \\
\text { em } \boldsymbol{m}\end{array}$ & $\begin{array}{l}\text { Porte-bruto (DWT, } \\
\text { dead-weight) em } \\
\text { ton }\end{array}$ & $\begin{array}{l}\text { Capacidade } \\
\text { em } \boldsymbol{m}^{3}\end{array}$ & $\begin{array}{l}\text { Aluguel do TCP, } \\
\text { hire (US\$ } / \text { dia) }\end{array}$ \\
\hline Small & $6,1-9,1$ & $15,6-23,4$ & $101,0-147,8$ & $<20.000$ & 18.500 & 10.500 \\
Handysize & $9,7-11,2$ & $24,6-28,8$ & $157,9-181,5$ & $20.000-40.000$ & 32.000 & 13.400 \\
Middle Range & $11,3-12,3$ & $31,4-32,2$ & $181,2-183,0$ & $40.000-50.000$ & 50.000 & 15.000 \\
\hline
\end{tabular}

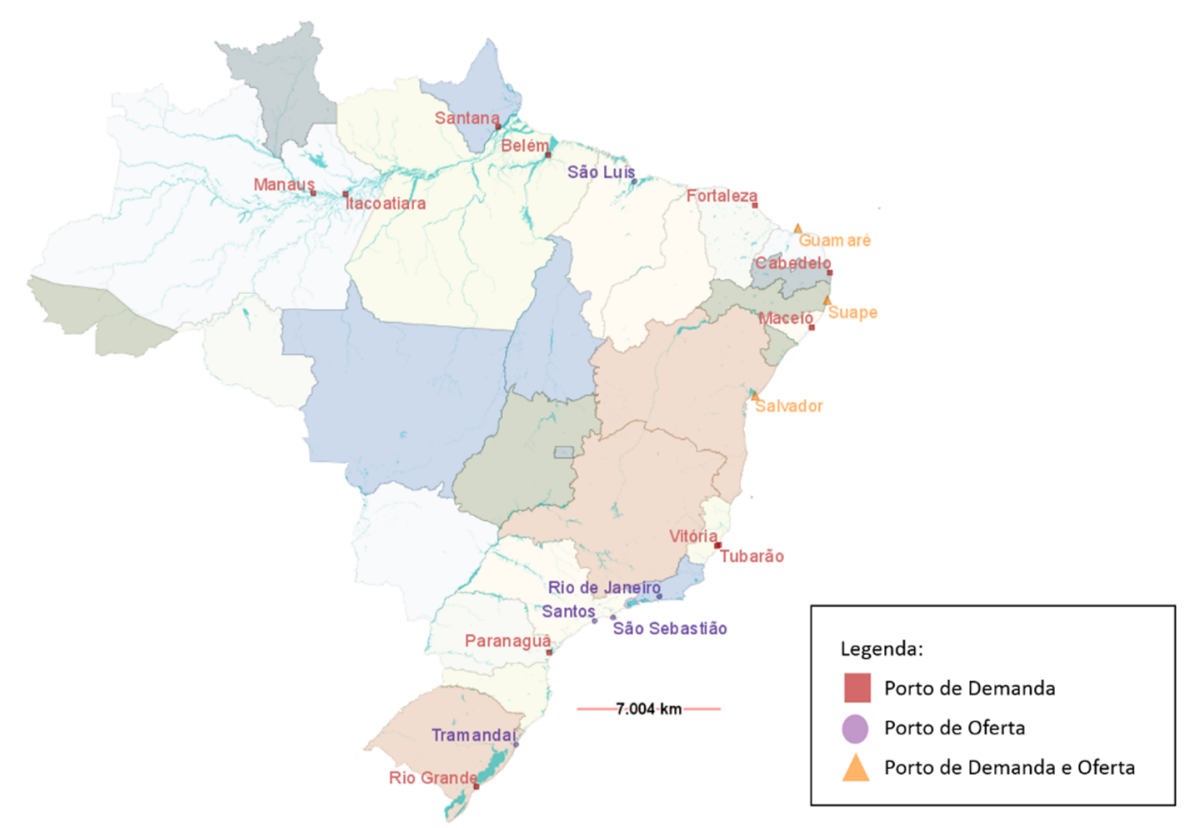

Figura 1: Mapa com a localização geográfica dos terminais

O calado dos tipos de navios e as restrições de profundidade foram utilizados para determinar os nós que permitem a operação de cada um dos tipos de navios. A Tabela 5 apresenta, as características (restrições operacionais e calado máximo para cada classe de navio) dos terminais apresentados na Figura 1.

A maioria dos terminais pode ser atendido por todas as classes de navios deste estudo, ainda que não seja com a carga máxima, como por exemplo o porto de Belém aonde os navios Handsize e Middle Range (MR) só podem operar com no máximo $20.000 \mathrm{~m}^{3}$ de volume. Entretanto, alguns portos não permitem operações de determinados tipos de petroleiros. Por exemplo, os portos de Tubarão e Vitória não aceitam navios da classe $M R$ ou superiores devido a restrições de calado. Já os portos de Guamaré e Tramandaí, de acordo com restrições da empresa estudada não permitiam navios de pequeno porte como Small e Handysize.

Para o modelo em questão, o tempo de viagem é definido como o tempo total entre a chegada do navio no porto de carga e o retorno a este mesmo porto. Este tempo foi estimado através de uma planilha de Excel que leva em consideração os tempos médios observados na prática para diversas etapas do ciclo de viagem, as distâncias entre os portos e uma velocidade média de 13 nós para todos os portes de navios. Já o horizonte de planejamento considerado foi de sessenta dias. Este período foi escolhido por abranger todos os tempos de viagem possíveis. 
Tabela 5: Restrições operacionais e calado máximo dos portos para os tipos de navios (Fonte: autores)

\begin{tabular}{|c|c|c|c|c|c|c|}
\hline \multirow{3}{*}{$\begin{array}{l}\text { Portos } \\
\text { Belém }\end{array}$} & \multicolumn{6}{|c|}{ Navios que operam no terminal / Calado Máximo ( $m 3$ ) } \\
\hline & \multicolumn{2}{|c|}{ Small } & \multicolumn{2}{|c|}{ Handysize } & \multicolumn{2}{|c|}{ Middle Range } \\
\hline & $\mathrm{V}$ & 18.500 & $\mathrm{v}$ & 20.000 & $\mathrm{~V}$ & 20.000 \\
\hline Cabedelo & v & 18.500 & v & 32.000 & $v$ & 35.000 \\
\hline Fortaleza & v & 18.500 & v & 32.000 & $\sqrt{ }$ & 36.000 \\
\hline Guamaré & $x$ & - & $x$ & - & 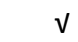 & 20.000 \\
\hline Itacoatiara & v & 18.500 & V & 32.000 & $v$ & 42.000 \\
\hline Maceió & v & 18.500 & v & 32.000 & $v$ & 35.000 \\
\hline Manaus & v & 18.500 & v & 32.000 & v & 42.000 \\
\hline Paranaguá & $\mathrm{v}$ & 18.500 & $\mathrm{v}$ & 32.000 & $\mathrm{~V}$ & 42.000 \\
\hline Rio de Janeiro & v & 18.500 & v & 27.000 & $v$ & 50.000 \\
\hline Rio Grande & v & 18.500 & V & 32.000 & $\sqrt{ }$ & 50.000 \\
\hline Salvador & $\mathrm{V}$ & 18.500 & $\mathrm{v}$ & 32.000 & $\mathrm{~V}$ & 50.000 \\
\hline Santana & v & 18.500 & v & 32.000 & v & 45.000 \\
\hline Santos & v & 18.500 & v & 32.000 & $v$ & 42.000 \\
\hline São Luís & $v$ & 18.500 & v & 32.000 & $v$ & 50.000 \\
\hline São Sebastião & v & 18.500 & v & 32.000 & $v$ & 50.000 \\
\hline Suape & v & 18.500 & v & 32.000 & $\sqrt{ }$ & 50.000 \\
\hline Tramandaí & $x$ & - & $x$ & - & $v$ & 50.000 \\
\hline Tubarão & $\mathrm{v}$ & 18.500 & $\mathrm{v}$ & 32.000 & $x$ & - \\
\hline Vitória & $\mathrm{v}$ & 18.500 & $\mathrm{v}$ & 25.000 & $x$ & - \\
\hline
\end{tabular}

Os custos operacionais foram calculados utilizando os custos dos combustíveis (bunker e marine gasoil) e as despesas portuárias. 0 custo dos combustíveis entre cada nó foi calculado multiplicando o consumo médio dos navios de cada porte que já fazem parte da frota pelo custo de combustível (dado de cenário). Por sua vez, os custos portuários foram obtidos dos sistemas de informação da companhia. 0 custo dos contratos Time Charter Party (TCP) utilizados no modelo foi calculado pela média dos custos de cada porte de navios já contratados pela empresa à época de elaboração deste trabalho, sendo destacados na Tabela 4.

Os dados dos cenários são aqueles que variam ao longo do tempo, em função, principalmente, de alterações na conjuntura econômica nacional e mundial. Neste sentido, é importante que sejam atualizados a cada rodada do modelo. Os principais dados dos cenários presentes no modelo são o suprimento e a demanda. Neste estudo, foram testados os seguintes cenários:

- Cenário 1: Provável, parâmetros originais estimados pela empresa;

- Cenário 2: Alta de $+2 \%$ na demanda de todos os produtos;

- Cenário 3: Alta de 5\% na demanda de Diesel);

- Cenário 4: Parada na produção de Diesel S500 em na refinaria que atende o terminal de Santos, perda de $50.000 \mathrm{~m} 3$ de oferta do produto neste terminal.

No Cenário 1, a oferta e a demanda de derivados em cada um dos portos foram estimadas através de dados da companhia, mensalmente revisados. Este cenário representa as estimativas originalmente previstas pela empresa para o período. Os volumes, em metros cúbicos, para este cenário são apresentados na Tabela 6.

O Cenário 2 foi feito após uma avaliação de dados mercadológicos da empresa que indicou a existência de uma variabilidade média mensal de entre $1 \%$ e $2 \%$ nas demandas previstas e realizadas para todos os derivados claros, entre os meses de agosto de 2013 e julho de 2014. Para verificar o comportamento do modelo em uma situação crítica de suprimento, foi proposto, que no Cenário 2, os dados de demanda de todos os produtos fossem elevados em $2 \%$. Como não foi considerado gerenciamento de estoque, os dados de suprimento também serão acrescidos no mesmo patamar. Em situações reais de aumento de demanda, seria necessário reduzir os estoques de refinarias e terminais ou comprar uma carga de importação não prevista. No entanto, esta variação média não representa adequadamente as variações extremas que podem ocorrer. Por exemplo, em fevereiro de 2014, a variação entre o mercado previsto e realizado de Diesel foi de 4,9\%, sendo esta a maior do período analisado. Por tal motivo, um 
terceiro cenário foi proposto variando somente a demanda e o suprimento de Diesel em 5\%. As paradas não programadas em unidades de produção das refinarias são os principais fatores para que a produção varie ao longo do tempo e afetam todo o perfil de processamento e produção da refinaria. Por isso, foi adicionado um quarto cenário simulando uma parada de 12 dias na unidade de fabricação do Diesel S500 de uma refinaria interligada ao Terminal de Santos e a consequente perda de produção de 50.000 $\mathrm{m}^{3}$ deste produto. A Figura 2 resume graficamente os diferentes cenários testados.

Tabela 6: Oferta (+) e Demanda (-) de produtos claros, em $m^{3}$, para o Cenário 1

\begin{tabular}{|c|c|c|c|c|c|c|c|}
\hline \multirow{2}{*}{ Terminal } & \multicolumn{7}{|c|}{ Produto } \\
\hline & Booster & Diesel ATE & Diesel S10 & Diesel S500 & Gasolina & Nafta & QAV \\
\hline Belém & & & $(76.000)$ & $(98.000)$ & (93.900) & & $(24.000)$ \\
\hline Cabedelo & & & & $(57.000)$ & $(91.800)$ & & \\
\hline Fortaleza & & & $(105.000)$ & $(47.000)$ & $(146.900)$ & & $(38.000)$ \\
\hline Guamaré & $(68.000)$ & 53.000 & $(56.000)$ & & & & \\
\hline Itacoatiara & & $(80.000)$ & (18.500) & $(66.000)$ & $(36.320)$ & & \\
\hline Maceió & & & & $(45.500)$ & $(44.890)$ & & \\
\hline Manaus & (132.000) & $(140.000)$ & $(112.500)$ & (109.500) & & & \\
\hline Paranaguá & & & $(202.000)$ & & & & \\
\hline Rio de Janeiro & 132.000 & & & & & 246.000 & \\
\hline Rio Grande & & & $(110.000)$ & & & & \\
\hline Salvador & 68.000 & & $(52.000)$ & 205.000 & 190.000 & $(246.000)$ & \\
\hline Santana & & & & $(65.000)$ & $(21.830)$ & & \\
\hline Santos & & & & 130.000 & 400.000 & & \\
\hline São Luís & & & 312.000 & 137.000 & 76.030 & & $(26.000)$ \\
\hline São Sebastião & & & 312.000 & 190.000 & & & \\
\hline Suape & & & 156.000 & (125.000) & $(77.400)$ & & \\
\hline Tramandaí & & (167.000) & & 90.000 & 72.000 & & \\
\hline Tubarão & & & $(26.000)$ & $(87.000)$ & (57.190) & & \\
\hline Vitória & & & $(22.000)$ & $(55.000)$ & (59.450) & & \\
\hline
\end{tabular}

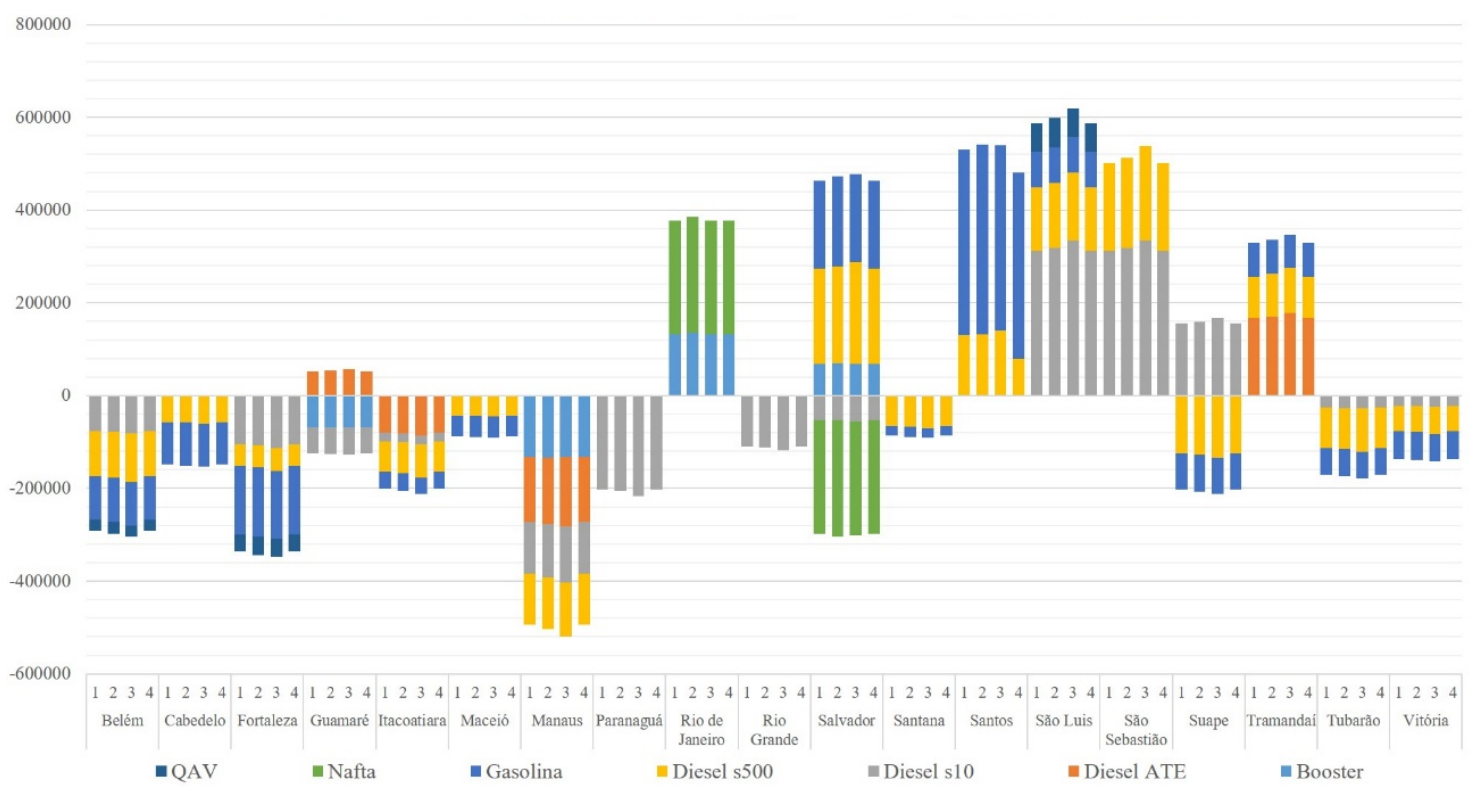

Figura 2: Balanço oferta-demanda $\left(\mathrm{m}^{3}\right)$ de cada produto para cada terminal e cenário.

\subsection{Descrição e interpretação dos resultados}

Para solucionar os cenários apresentados na seção 5.1, o modelo foi executado em um computador com processador Intel ${ }^{\circledR} \mathrm{Core}^{\mathrm{T}}$ i5-4440 CPU 3.10GHz com memória RAM de 8,00 GB. A plataforma de modelagem escolhida para solucionar o problema foi o $A I M M S^{\circledR}$ e dois grandes solvers de programação linear 
inteira-mista foram testados para otimizar o problema: CPLEX v.12.6.2 e Gurobi v.6.5. Os melhores resultados computacionais foram obtidos com o CPLEX, que atingiu a solução ótima do Cenário 1 em um tempo de 1.712,91 segundos. Já o Gurobi precisou de 4.817,11 segundos para atingir o mesmo resultado. A seguir, os resultados do cenário 1 são detalhados e analisados.

0 primeiro resultado do Cenário 1 apresentado é o número de viagens realizadas nos arcos de movimentação, que é representado pelas variáveis de decisão $x_{v i j}$. A Tabela 7 traz as viagens alocadas aos navios da classe Small.

Tabela 7: Número de viagens nos arcos de movimentação atendidas por navios Small (Fonte: autores)

\begin{tabular}{llllll}
\hline Origem $\boldsymbol{i} \backslash$ Destino $\boldsymbol{j}$ & Belém & Fortaleza & Suape & Tubarão & Vitória \\
\hline São Luís & 14 & 1 & - & - & - \\
Salvador & 2 & - & 1 & - & - \\
Santos & - & - & - & 1 & 1 \\
\hline
\end{tabular}

A Tabela 7 revela que os navios de menor porte (Small), são alocados preferencialmente para o suprimento de Belém, porto com a menor profundidade. Sendo assim, é percebida uma vantagem em alocar esta classe de navio a este porto, e assim aproveitar sua capacidade integral, uma vez que as outras classes somente podem operar em Belém se estiverem aliviados. Além disso, os navios Small também são alocados para complementar o mercado de outros portos que são atendidos prioritariamente por navios maiores, como Fortaleza, Suape, Tubarão e Vitória. Os navios de porte intermediário (Handysize) também foram prioritariamente alocados para atender portos onde podem operar em plena capacidade, como Fortaleza, Cabedelo, Maceió, Tubarão e Vitória. As indicações de três viagens para Manaus e duas para Rio Grande são feitas para complementar o atendimento do mercado destes portos. A Tabela 8 apresenta um filtro somente das viagens alocadas a esta classe de navio.

Os maiores navios (Middle Range, $M R$ ) foram prioritariamente alocados em arcos onde, devido a restrições nos portos de origem e/ou destino, somente eles estão aptos a operar, como Guamaré e Tramandaí. Verifica-se também a indicação de os utilizar em arcos onde existe grande movimentação de produto e pouca ou nenhuma restrição de calado, como Rio de Janeiro - Salvador e São Sebastião - Paranaguá. Além disso, ressalta-se que esta classe de navios também foi alocada para realizar viagens longas, como São Sebastião - Manaus e São Sebastião - Itacoatiara. As viagens da classe de navios $M R$ são apresentadas na Tabela 9.

Tabela 8: Número de viagens nos arcos de movimentação com navios Handysize (Fonte: autores)

\begin{tabular}{llllllllll}
\hline Origem $\boldsymbol{i} \backslash$ Destino $\boldsymbol{j}$ & Manaus & Itacoatiara & Fortaleza & Cabedelo & Maceió & Salvador & Tubarão & Vitória & Rio Grande \\
\hline São Luís & - & 2 & 4 & - & - & - & - & - & - \\
Suape & - & - & - & - & - & - & 2 & 1 & 1 \\
Salvador & - & - & 4 & 3 & 3 & - & - & - & - \\
Rio de Janeiro & 2 & - & - & - & - & - & - & - & - \\
São Sebastião & 1 & - & - & - & - & - & - & 1 & 2 \\
Santos & - & - & 2 & 2 & 2 & - & 4 & 3 & - \\
\hline
\end{tabular}

É importante perceber que, em alguns momentos, a movimentação entre portos distantes se faz necessária para obter um ganho maior em outra frente de movimentação. 0 segundo resultado do Cenário 1 apresentado pelo modelo é o volume total de cada um dos produtos transportados nos arcos de movimentação, para cada um dos portes de navios, representada pela variável de decisão $y_{v i j p}$. A melhor maneira de interpretar este resultado é analisando a distribuição de produto a partir de cada fonte de suprimento. A Tabela 10 apresenta os volumes movimentados a partir de Tramandaí.

Para analisar os dados da Tabela 10, é preciso levar em consideração que só existe demanda de Diesel ATE em Manaus e Itacoatiara. Ao observar a Tabela 9, constata-se que o modelo aloca três viagens de Tramandaí para Manaus e na terceira viagem, onde haveria uma praça disponível de $22.000 \mathrm{~m}^{3}$, o modelo aproveita para movimentar Diesel S500. 0 mesmo tipo de raciocínio aplica-se a Itacoatiara. Nas 
outras viagens de Tramandaí para Santana e Suape, o modelo compartilha as cargas entre Gasolina e Diesel $S 500$ no intuito de aproveitar a máxima capacidade do navio. Como observado, as movimentações realizadas a partir de Tramandaí afetam diretamente as movimentações realizadas a partir de Guamaré, onde o Diesel ATE é o único produto excedente. Verifica-se que o modelo utiliza a oferta de Guamaré para complementar o saldo de mercado a ser entregue em Manaus e Itacoatiara. A próxima fonte de suprimento a ser analisada será Santos, e o volume movimentado a partir deste porto é apresentado na Tabela 11.

Tabela 9: Número de viagens nos arcos de movimentação com navios $M R$ (Fonte: autores)

\begin{tabular}{lllllllll}
\hline Origem $\boldsymbol{i} \backslash$ Destino $\boldsymbol{j}$ & Manaus & Itacoatiara & Santana & Guamaré & Suape & Salvador & Paranaguá & Rio Grande \\
\hline São Luís & 3 & - & - & - & - & - & - & - \\
Guamaré & 2 & 1 & - & - & - & - & - & - \\
Suape & - & - & - & 3 & - & - & - & - \\
Salvador & - & - & - & - & - & - & - & - \\
Rio de Janeiro & 2 & - & - & - & - & 5 & - & - \\
São Sebastião & 1 & 1 & - & - & 1 & - & 5 & 1 \\
Santos & - & - & - & - & 2 & - & - & - \\
Tramandaí & 3 & 2 & 1 & - & 1 & - & - & - \\
\hline
\end{tabular}

Tabela 10: Volume movimentado $\left(\mathrm{m}^{3}\right)$ a partir de Tramandaí e Guamaré (Fonte: autores)

\begin{tabular}{lllllll}
\hline \multirow{2}{*}{ Origem $\boldsymbol{i}$} & \multicolumn{2}{c}{ Destino $\boldsymbol{j}$} & Manaus & Itacoatiara & Santana & \multirow{2}{*}{ Suape } \\
\cline { 2 - 6 } & Produto $p$ & Navio $v$ & & & & \\
\multirow{3}{*}{ Tramandaí } & Gasolina & $M R$ & - & 21.000 & 21.830 & 4.180 \\
& DiesekI S500 & $M R$ & 22.000 & - & 22.180 & 45.820 \\
& Diesel ATE & $M R$ & 104.000 & 63.000 & - & - \\
\hline Guamaré & Diesel ATE & $M R$ & 36.000 & 17.000 & - & - \\
\hline
\end{tabular}

Tabela 11: Volume movimentado a partir de Santos (Fonte: autores)

\begin{tabular}{|c|c|c|c|c|c|c|c|}
\hline \multirow{2}{*}{ Origem $i$} & \multicolumn{2}{|c|}{ Destino $j$} & \multirow{2}{*}{ Fortaleza } & \multirow{2}{*}{ Cabedelo } & \multirow{2}{*}{ Suape } & \multirow{2}{*}{ Vitória } & \multirow{2}{*}{ Tubarão } \\
\hline & Navio $v$ & Produto $p$ & & & & & \\
\hline \multirow{5}{*}{ Santos } & Small & Gasolina & - & - & - & 18.500 & 18.500 \\
\hline & \multirow{2}{*}{ Handysize } & Gasolina & 62.780 & 64.000 & - & 40.950 & 38.690 \\
\hline & & Diesel 5500 & 1.220 & - & - & 31.000 & 87.000 \\
\hline & \multirow{2}{*}{$M R$} & Gasolina & - & - & 73.220 & - & - \\
\hline & & Diesel $S 500$ & - & - & 10.780 & - & - \\
\hline
\end{tabular}

A análise dos dados da Tabela 11 permite concluir que os pólos atendidos pela oferta de Santos são alocados em função da menor distância. Após atender integralmente o mercado de Gasolina e Diesel S500 de Tubarão, o modelo segue alocando produto para Vitória, Suape, Cabedelo e Fortaleza, exatamente na ordem crescente de distância, até esgotar todo o volume disponível. Além disso, novamente é indicado o compartilhamento de dois produtos em uma mesma viagem, neste caso Gasolina e Diesel S500 para Fortaleza e Suape, com o intuito de aproveitar a máxima capacidade do navio.

É importante observar que o fato de o porto de Santos poder operar as três classes de navios gera uma flexibilidade na escolha dos destinos, facilitando, assim, a alocação do produto disponível em função da menor distância. Este mesmo padrão do modelo foi observado nos outros portos de carga. Neles, o modelo buscou alocar viagens em função da menor distância, realizar o menor número de viagens com a máxima capacidade possível nos navios e compartilhar as cargas, levando em consideração as restrições de calado em cada terminal.

0 último resultado do cenário 1 apresentado pelo modelo é o número total de navios de cada porte que deverá conter na frota para que todas as restrições sejam atendidas, variável de decisão $z_{v}, 0$ modelo determinou uma frota ótima de 4 navios do tipo Small, 9 navios do tipo Handysize e 13 navios do tipo Middle Range (MR). Esta frota ótima foi capaz de atender as demandas de todos os pólos consumidores 
e a oferta da produção excedente das refinarias no horizonte de planejamento analisado. 0 custo total, durante o horizonte de planejamento, da frota ótima foi de US\$44.672.258,00.

No ano de 2014 (mesmo ano dos meses analisados), a equipe da empresa dimensionou um total de dois navios Small, seis navios Handysize e vinte e três navios Middle Range para o atendimento da cabotagem de derivados claros. 0 cenário 1 foi novamente executado fixando a frota disponibilizada pela empresa no período (ou seja, fixando a variável $z_{v}$ ), o modelo obteve um custo estimado dessa frota de US\$50.399.987,00. Partindo da premissa que o programador de navios possui essa frota de 33 navios já dimensionada à disposição, os resultados do modelo ajudam-no a prever os momentos de ociosidade ou de alta utilização da frota. No horizonte analisado, o modelo iria indicar uma frota ótima de 26 navios, permitindo com que o programador pudesse liberar até 7 navios para afretamento ou viagens no longo curso, diminuindo a ociosidade da frota e aumentando a eficiência da mesma. 0 modelo proposto, então, permitiria gerar economias no curto prazo para a empresa.

Com o intuito de analisar a robustez das soluções geradas pelo modelo frente a variações nos seus principais parâmetros (oferta e demanda nos terminais). Os autores compararam os resultados do Cenário 1 com os outros três cenários apresentados na seção 5.1. Os resultados desta análise de cenários estão apresentados na Tabela 12.

Tabela 12: Número total de navios de cada porte para cada um dos cenários (Fonte: autores)

\begin{tabular}{lllll}
\hline Análise de Cenários & $\begin{array}{l}\text { Cenário 1: Previsão } \\
\text { Classe de Navio } \boldsymbol{v}\end{array}$ & $\begin{array}{l}\text { Cenário 2: Demanda } \\
\mathbf{+ 2 \%}\end{array}$ & $\begin{array}{l}\text { Cenário 3: Demanda } \\
\text { Diesel +5\% }\end{array}$ & $\begin{array}{l}\text { Cenário 4: Parada na Produção } \\
\text { Diesel S500 na refinaria }\end{array}$ \\
\hline Small & 4 & 3 & 5 & 3 \\
Handysize & 9 & 10 & 9 & 8 \\
Middle Range & 13 & 13 & 13 & 14 \\
\hline
\end{tabular}

Analisando a Tabela 12, percebe-se que para uma variabilidade de $2 \%$ na demanda, a frota dimensionada apresentou poucas alterações em relação à frota original. 0 modelo indica apenas a necessidade de mais um Handysize e corta um Small. Portanto, a frota original é aderente a uma variação de mercado de $2 \%$ para todos os produtos. A frota original também foi aderente para um pico de $5 \%$ na demanda de Diesel, acrescentando-se apenas um navio Small na frota. Para o cenário de redução na produção de Diesel $S 500$ da RPBC observaram-se novamente poucas as alterações no tamanho da frota. 0 modelo retira um navio Small e um Handysize e adiciona um $M R$ na frota original. Em todos os cenários, o modelo se adequou e continuou priorizando as movimentações entre os portos em função da menor distância. Destaca-se que a solução do Cenário 1 (original) foi aderente com as soluções dos outros cenários testados, evidenciando a robustez do modelo para variações na demanda e oferta dos terminais.

\section{CONSIDERAÇÕES FINAIS}

O transporte marítimo é de fundamental importância para as atividades da indústria petrolífera e está inserido em um ambiente permeado de flutuações e incertezas, sendo o dimensionamento da frota uma das principais decisões estratégicas a serem tomadas. Neste sentido, este trabalho teve por objetivo propor um modelo matemático para o dimensionamento da frota dos navios de derivados claros para cabotagem da empresa de petróleo estudada, adaptando um modelo apresentado por Steffensen (2012), onde a função objetivo minimiza os custos totais da frota dimensionada. A plataforma de modelagem utilizada foi o software $A I M M{ }^{\circledR}$ e o solver utilizado foi o $C P L E X$ v.12.6. Os resultados obtidos no cenário original reduziram os custos estimados da frota em 11.36\%, gerando economias de milhões de dólares. A minimização dos custos totais induz o modelo a maximizar o aproveitamento das capacidades totais dos navios. Viagens onde esta capacidade não seja inteiramente utilizada são evitadas ao máximo e busca-se o compartilhamento de diversos produtos a bordo de um mesmo navio. 0 modelo procura, sempre que possível, atender um polo consumidor a partir da fonte de suprimento mais próxima. E, além de dimensionar a quantidade de navios, o modelo indica qual o porte de cada um. 
Para verificar a aderência da frota dimensionada diante variações na produção e na demanda dos derivados, foram realizadas três análises de cenários - variando a demanda de todos os produtos em $+2 \%$ (Cenário 2), variando a demanda de Diesel em $+5 \%$ (Cenário 3) e reduzindo a produção de Diesel em uma refinaria (Cenário 4). Em todos os casos, a frota dimensionada originalmente mostrou-se aderente, apresentando poucas variações na quantidade e nos portes dos navios. Após esta análise, os programadores estarão aptos a prever com maior precisão os momentos de ociosidade ou de alta utilização da frota já contratada pela empresa, durante um horizonte de planejamento pré-estabelecido. Nos casos em que a frota estiver ociosa, um ou mais navios poderão ser programados para carregarem produtos no exterior, evitando assim a contratação de um navio em Voyage Charter Party para este fim, gerando a economia de milhares de dólares. Como atualmente, na empresa estudada, o dimensionamento e a programação dos navios são executados sem modelos matemáticos, a aplicação do modelo aqui proposto facilitará o processo de construção de cenários, dando visibilidade a soluções pouco usuais, gerando maior interatividade entre os programadores e potencializando economias.

Durante a elaboração deste trabalho, diversos temas referentes à programação de navios foram estudados e observaram-se diversas oportunidades de pesquisas futuras. A principal delas refere-se à aplicação de modelos de programação e roteamento dos navios em função dos níveis de estoques nos portos de origem e destino. Em relação ao dimensionamento de frota, o emprego da simulação pode contribuir com resultados satisfatórios. Também é importante, a elaboração de um modelo mais robusto com um horizonte de planejamento mais longo, novas variáveis e a inserção de incerteza através de otimização estocástica ou robusta. Espera-se que este trabalho contribua para a realização de pesquisas futuras.

\section{REFERÊNCIAS BIBLIOGRÁFICAS}

Bakkehaug, Rikard; E. S. Eidem; K. Fagerholt e L. M. Hvattum (2014) A Stochastic Programming Formulation for Strategic Fleet. Transportation Research Part E: Logistics and Transportation Review, v. 72, p. 60-76.

Brown, G. G.; G. W. Graves e D. Ronen (1987) Scheduling Ocean Transportation of Crude Oil. Management Science, v. 33, n. 3, p. 335-346. DOI: $10.1287 /$ mnsc.33.3.335

Christiansen, M.; K. Fagerholt e D. Ronen (2004) Ship Routing and Scheduling: Status and Perspectives. Transportation Science, v. 38, n. 1, p. 1-18. DOI: $10.1287 /$ trsc.1030.0036

Christiansen, M.; K. Fagerholt; B. Nygreen e D. Ronen (2007) Chapter 4 Maritime Transportation. In: Barnhart, C. e G. Laporte (eds.) Handbooks in Operations Research and Management Science, Transportation, v. 14, p.189-284. D0I: 10.1016/S09270507(06)14004-9

Christiansen, M.; K. Fagerholt; B. Nygreen e D. Ronen (2013) Ship Routing and Scheduling in the New Millennium. European Journal of Operational Research, v. 228, n. 3, p. 467-483. DOI: 10.1016/j.ejor.2012.12.002

CNT (2016) Anuário CNT do Transporte: Estatísticas consolidadas - Transporte Aquaviário. Confederação Nacional do Transporte, Brasília, DF.

Dantzig, G. B. e D. R. Fulkerson (1954) Minimizing the Number of Tankers to Meet a Fixed Schedule. Naval Research Logistics Quarterly, v. 1, n. 3, p. 217-222.

Diz, G. S. D. S.; F. Oliveira e S. Hamacher (2016) Improving maritime inventory routing: application to a Brazilian petroleum case. Maritime Policy \& Management, v.44, n.1, p. 42-61. DOI: 10.1080/03088839.2016.1216622

Gundegjerde, C.; I. B. Halvorsen; E. E. Halvorsen-Weare; L. M. Hvattum e L. M. NonåS (2015) A Stochastic Fleet Size and Mix Model for Maintenance Operations at Offshore Wind Farms. Transportation Research, Part C, v. 52, p. 74-92. DOI: 10.1016/j.trc.2015.01.005

Hoff, A.; H. Anderson; M. Christiansen; G. Hasle e A. Lokketangen (2010) Industrial Aspects and Literature Survey: Fleet Composition and Routing. Computers \& Operations Research, v. 37, n. 12, p. 2041-2061. DOI: 10.1016/j.cor.2010.03.015

Mulder, J. e R. Dekker (2014) Methods for Strategic Liner Shipping Network Design. European Journal of Operational Research, v. 235, n. 2, p. 367-377. DOI: 10.1016/j.ejor.2013.09.041

Nunes, P. M.; F. Oliveira; S. Hamacher; P. Hamacher; W. Teixeira e F. Munck (2010) Análise do Planejamento de Abastecimento da Cadeia de Petróleo no Brasil. Rio Oil \& Gas Expo and Conference 2010, IBP, Rio de Janeiro, RJ.

Pantuso, G.; K. Fagerholt e L. M. Hvattum (2013) A Survey on Maritime Fleet Size and Mix Problems. European Journal of Operational Research, v. 235, n. 2, p. 341-349. DOI: 10.1016/j.ejor.2013.04.058

Pelizaro, C. (2008) Dimensionamento de Frota em uma Empresa de Petróleo Integrada. Dissertação (Mestrado em Engenharia Industrial) - PUC-Rio. Rio de Janeiro, RJ, junho de 2008.

Rocha, M. R. S. (2012) Distribuição de Petróleo no Mercado Internacional: Um Instrumento de Suporte à Decisão Baseado em Estudo de Caso Prático. Dissertação (Mestrado Profissional em Engenharia de Produção) - PUC-Rio. Rio de Janeiro, RJ, novembro de 2012.

Ronen, D. (1983) Cargo Ships Routing and Scheduling: Survey of Models and Problems. European Journal of Operational Research, v. 12, n. 2, p. 119-126. DOI: 10.1016/0377-2217(83)90215-1 
Ronen, D. (1993) Ship Scheduling: the Last Decade. European Journal of Operational Research, v. 71, n. 3, p. 325-333. DOI: 10.1016/0377-2217(93)90343-L

Saraceni, P. P. (2006) Transporte Marítimo de Petróleo e Derivados. Editora Interciência, Rio de Janeiro.

Steffensen, M. A. (2012) Maritime Fleet Size and Mix Problems: an Optimization Based Modeling Approach. Dissertação (Mestrado em Marine Technology) - NTNU. Trondheim, Norway, junho de 2012.

UNCTAD (2016) Review of Maritime Transport: The long-term growth prospects for seaborne trade and maritime businesses. United Nations Conference on Trade and Development, United Nations, Geneva, Switzerland.

Xinlian, X.; L. Shu-Fan; J. Zhuo-Shang e C. Shengfu (1989) Study and Application on the Linear Model of Fleet Planning. Shipbuilding of China, v. 3, p. 59-66.

Xinlian, X.; W. Tengfei e C. Daisong (2000) A Dynamic Model and Algorithm for Fleet Planning. Maritime Policy \& Management, v. 27, n. 1, p. 53-63. DOI: $10.1080 / 030888300286680$

Zeng, Q. e Z. Yang (2007) Model Integrating Fleet Design and Ship Routing Problems for Coal Shipping. In: Shi, Y.; G. D. van Albada; J. Dongarra e P. M. A. Sloot (eds.) Computational Science - ICCS 2007. Beijing, China. 